\title{
A non-linear random vibration reliability analysis method for multi degree of freedom helical gear driven system
}

\author{
Chang $\mathrm{Li}^{\mathrm{a}, *}$, Mingyong $\mathrm{Hu}^{\mathrm{b}}$ and Xing Han ${ }^{\mathrm{c}}$ \\ School of Mechanical Engineering \& Automation, University of Science and Technology Liaoning, \\ Anshan 114051, China. \\ alichang2323-23@163.com, b2277182899@qq.com, chanxinggctx@163.com
}

\begin{abstract}
Firstly, taking rigidity excitation, error excitation, meshing impact, clearance variety and other non-linear factors into account, the bend-twist-shaft-swing non-linear coupled vibration model and differential equations for the multi degree of freedom helical gear driven system are built based on the lumped mass method. Secondly, the model is solved by the fifth order variable step size and self-adapted method (Runge-Kutta method), and then the system vibration time-domains and frequency-domains are obtained. On the above basis, we fitted the system different random error's probability distributions and carried out sampling calculation by Monte Carlo method for the system, and then got the non-linear random structure system's vibration reliability at different time. This method is easy to program, and provides a theory basis for helical gear driven system's quantification design of controllable structure and performance.
\end{abstract}

Keywords: Multi degree of freedom helical gear driven system; Monte Carlo method; fifth order variable step size and self-adapted method; random vibration response.

\section{Introduction}

The helical gear driven system is widely used in machinery, metallurgy, automobile, aerospace, and other fields. Traditional engineering analysis uses the deterministic mechanical model, using the mean parameters instead of its actual value. When the variability of actual system is larger, the calculation results can produce deviation. The randomness of the actual system includes the following aspects: (1) the randomness of material characteristics; (2) the randomness of physical dimension; (3) the randomness of structure boundary conditions; (4) the randomness of physical property parameters[1-2]. Because of all kinds of random factors, the gear system itself has some random characters. Therefore, in the design of gear system, the system randomness is of great importance in engineering practice. Therefore, the random factors of actual engineering have a very significant impact on the helical gear driven system based on random vibration reliability analysis [3-5].

\section{Multi Degree of Freedom Non-linear Random System Reliability Analysis Method Based on Monte Carlo Method}

$\boldsymbol{X}$ is a set of random variables of influence the multi degree of freedom non-linear random system, $\boldsymbol{X}=\left[X_{1}, X_{2}, \ldots, X_{n}\right]^{\mathrm{T}}, X_{h}(h=1,2,3, \ldots, n)$ is the basic random quantity; $Y_{k}(k=1,2,3, \ldots, s)$ is the system performance output determined by operating requirements and the function of a random variable $\boldsymbol{X}$, namely:

$$
\begin{aligned}
& Y_{k}=f(\boldsymbol{X})=f\left(X_{1}, X_{2}, X_{3}, \cdots X_{n}\right) \\
& \left(z_{\text {klower range value }}, z_{\text {k upper range value }}\right) \text { is the allowable limit value of system output response, }(k=1,2,3, \ldots,
\end{aligned}
$$

$s)$. The system has $s$ kinds of failure modes. Therefore, there are $s$ kinds of limit equations of state in system. The required reliability of system performance output response under the first $k$ times failure mode is:

$$
R_{k}=P\left(z_{\text {klower rangevalue }} \leq Y_{k} \leq z_{\text {kupper rangevalue }}\right)(k=1,2,3, \ldots, s)
$$

The system reliability of $s$ kinds of failure modes under correlated conditions is: 


$$
R=P\left\{\prod_{k=1}^{s} R_{k}\right\}=P\left\{\prod_{k=1}^{s}\left[z_{k \text { lower range value }} \leq Y_{k} \leq z_{k \text { upper range value }}\right]\right\}
$$

The multi degree of freedom non-linear random structural system vibration reliability is solved based on the Monte Carlo method. The method is applicable to reliability calculation of any distribution random variables and any combination random variables. The calculation accuracy is improved with the increase of simulation times. The program layout of random vibration reliability solution is shown in Fig. 1.

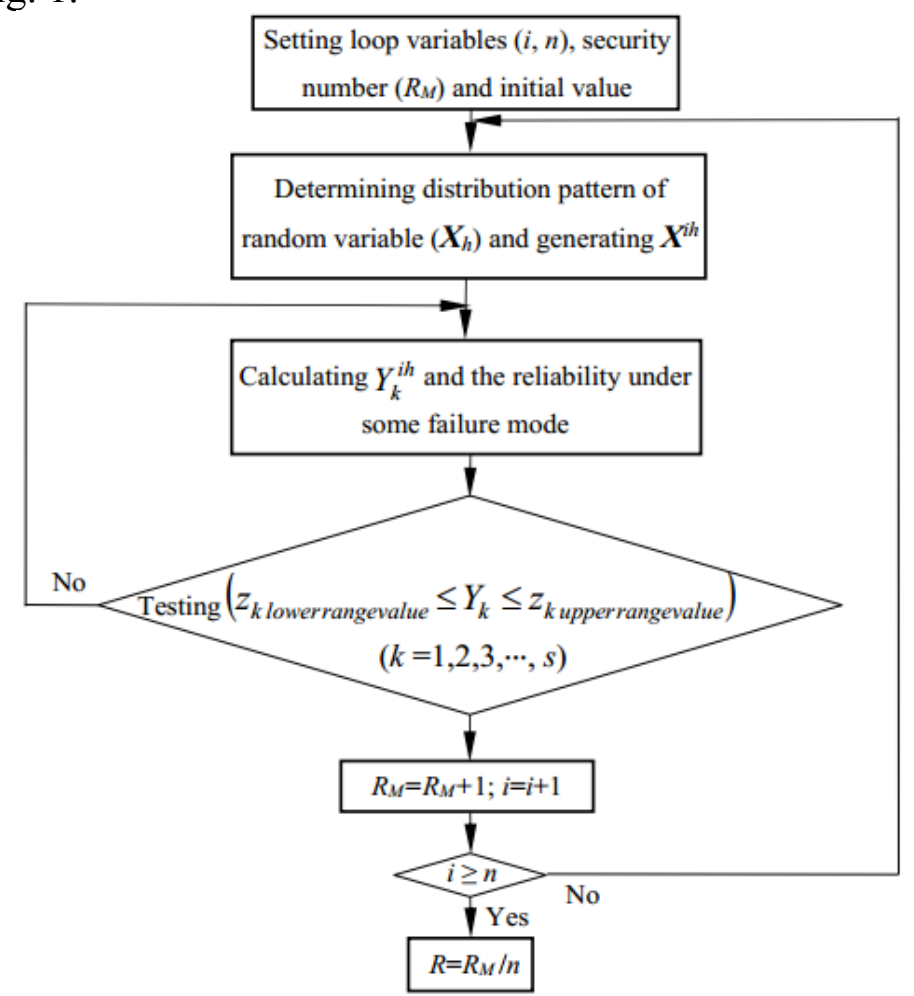

Fig. 1 Calculation process of system vibration reliability

\section{Building and Solving Bend-twist-axis-swing Non-linear Coupling Vibration Model for Helical Gear Driven System}

\subsection{Building the Model.}

The helical gear driven system has vibration of bending, twisting, axis and swing. Therefore, in order to study further the dynamic characteristics for helical gear driven system, we built its bend-twist-axis-swing non-linear coupling vibration model. The dynamic model is built based on the lumped mass method, as shown in Fig. 2. It is ignored that the tooth surface friction and the torsional elastic deformation of drive axle in calculation. The cross bending elastic deformation of drive axle and supporting is simulated by supporting spring of relative to the gear symmetrical arrangement ${ }^{[6]}$.

Each gear has five degrees of freedom: the $x, y$ direction of along gear wheel shaft, the axial $z$ direction, the $\theta_{z}$ direction, the $\theta_{x}$ direction. The spring and damping replace the stiffness and damping of bearing contact and tooth mesh. $c_{m}, k_{h}$ are respectively the damping and the time varying meshing stiffness value of gear pair; $k_{i j}(i=p, g ; j=x, y, z), c_{i j}(i=p, g ; j=x, y, z)$ are respectively the supporting stiffness and equivalent value of damping; $e(t)$ is the static error.

$\{\delta\}$ is the displacement matrix of dynamic model for helical gear driven system. $\{\delta\}$ is:

$\{\delta\}=\left\{x_{p}, y_{p}, z_{p}, \theta_{p x}, \theta_{p z}, x_{g}, y_{g}, z_{g}, \theta_{g x}, \theta_{g z}\right\}^{\mathrm{T}}$

Where, $x_{i}, y_{i}, z_{i}(i=p, g)$ are respectively the translational vibration displacement of center point of driven gear and driving gear along the $x, \mathrm{y}, z$ three directions; $\theta_{i x}(i=p, g)$ is the torsion pendulum vibration displacement of center point of driven gear and driving gear along the $x$ axis; $\theta_{i z}(i=p, g)$ is the torsional vibration displacement of driven gear and driving gear around the $z$ axis. 
The helix angle of driving gear is set to the $\beta . \alpha_{t}$ is the transverse pressure angle. The vibration relationships of contact points between $x, y$ and $z$ directions are respectively:

$$
x=y \cdot \tan \alpha_{t}=\frac{y \cdot \tan \alpha_{n}}{\cos \beta}, z=y \cdot \tan \beta
$$

The center point of driving gear is set to the $O_{p}$, the relationships between generalized displacement and vibration displacements of $O_{p}$ in contact points are:

$$
\left\{\begin{array}{l}
\bar{x}_{p}=x_{p}-\bar{y}_{p} \tan \alpha_{t}=x_{p}-\left(y_{p}+\theta_{p z} R_{p}\right) \tan \alpha_{t} \\
\bar{y}_{p}=y_{p}+\theta_{p z} R_{p} \\
\bar{z}_{p}=z_{p}+\bar{y}_{p} \tan \beta=z_{p}+\left(y_{p}+\theta_{p z} R_{p}\right) \tan \beta
\end{array}\right.
$$

The center point of driven gear is set to the $O_{g}$, the contact points displacement and vibration displacement are respectively:

$$
\left\{\begin{array}{l}
\bar{x}_{p}=x_{p}+\bar{y}_{p} \tan \alpha_{t}=x_{p}+\left(y_{p}-\theta_{p z} R_{p}\right) \tan \alpha_{t} \\
\bar{y}_{p}=y_{p}-\theta_{p z} R_{p} \\
\bar{z}_{p}=z_{p}+\bar{y}_{p} \tan \beta=z_{p}+\left(y_{p}-\theta_{p z} R_{p}\right) \tan \beta
\end{array}\right.
$$

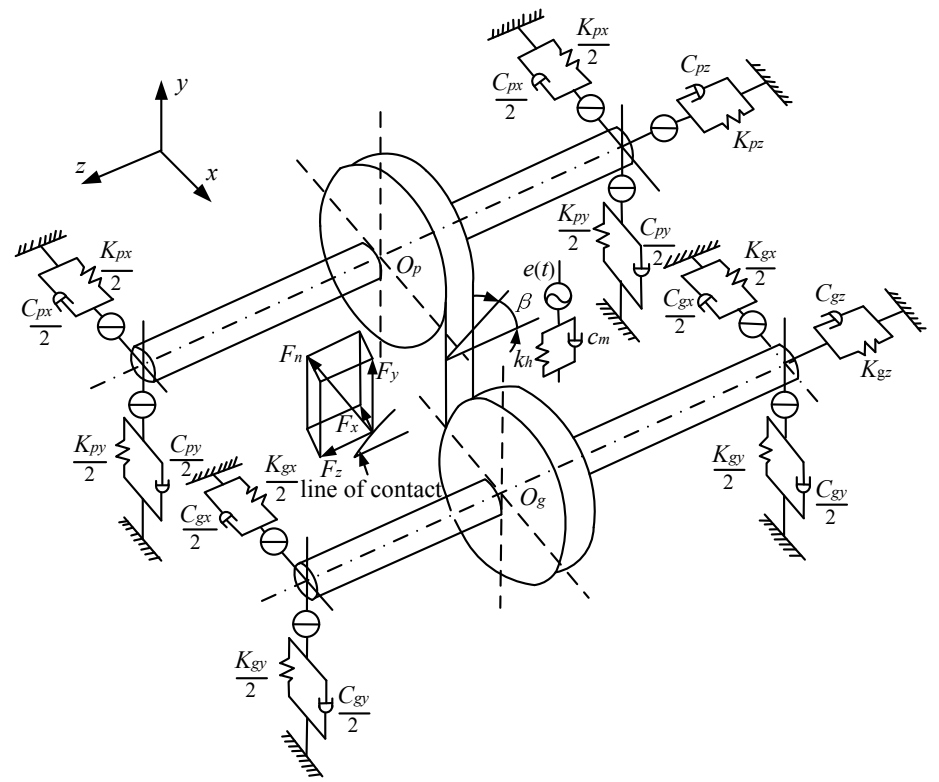

Fig. 2. Helical gear bend-twist-shaft-swing vibration analysis model

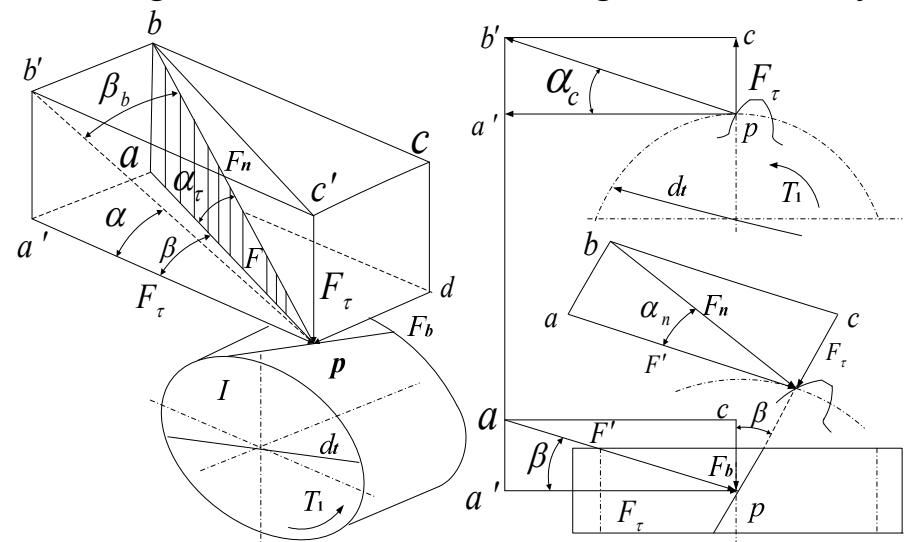

Fig. 3. Resolution graph of meshing force for helical gear

The resolution graph of meshing force is shown in Fig.3. $k_{h}$ is the time varying meshing stiffness of helical gear, $c_{m}$ is the meshing damping, $e$ is the meshing error, the direction vectors of $x, y$ and $z$ are respectively: 


$$
\left\{\begin{array}{lll}
k_{m x}=k_{h} \sin \alpha & k_{m y}=k_{h} \cos \alpha \cos \beta & k_{m z}=k_{h} \cos \alpha \sin \beta \\
c_{m x}=c_{m} \sin \alpha & c_{m y}=c_{m} \cos \alpha \cos \beta & c_{m z}=c_{m} \cos \alpha \sin \beta \\
e_{x}=e \sin \alpha & e_{y}=e \cos \alpha \cos \beta & e_{z}=e \cos \alpha \sin \beta
\end{array}\right.
$$

The elastic deformation expressed by vibration displacement is:

$$
\begin{aligned}
& x_{3}=\bar{x}_{p}-\bar{x}_{g}-e_{x}=x_{p}-x_{g}-\left(y_{p}+\theta_{p z} R_{p}+y_{g}-\theta_{g z} R_{g}\right) \tan \alpha_{t}-e_{x} \\
& y_{3}=\bar{y}_{p}-\bar{y}_{g}-e_{y}=y_{p}-y_{g}+\theta_{p z} R_{p}+\theta_{g z} R_{g}-e_{y} \\
& z_{3}=\bar{z}_{p}-\bar{z}_{g}-e_{z}=z_{p}-z_{g}+\left(y_{p}+\theta_{p z} R_{p}-y_{g}+\theta_{g z} R_{g}\right) \tan \beta-e_{z}
\end{aligned}
$$

The radial dynamic meshing force called $F_{x}$ is:

$$
\begin{aligned}
& F_{x}=k_{m x} f\left(x_{s}\right)+c_{m x} \dot{x}_{s}=k_{h} \sin \alpha \cdot f\left\lfloor x_{p}-x_{g}-\left(y_{p}+\theta_{p z} R_{p}+y_{g}-\theta_{g z} R_{g}\right) \tan \alpha_{t}-e_{x}\right\rfloor+ \\
& c_{m} \sin \alpha\left[\dot{x}_{p}-\dot{x}_{g}-\left(\dot{y}_{p}+\dot{\theta}_{p z} R_{p}+\dot{y}_{g}-\dot{\theta}_{g z} R_{g}\right) \tan \alpha_{t}-\dot{e}_{x}\right]
\end{aligned}
$$

The tangential dynamic meshing force called $F_{y}$ is:

$$
\begin{aligned}
& F_{y}=k_{m y} f\left(y_{s}\right)+c_{m y} \dot{y}_{s}=k_{h} \cos \alpha \cdot \cos \beta \cdot f\left\lfloor y_{p}-y_{g}+\theta_{p z} R_{p}+\theta_{g z} R_{g}-e_{y}\right\rfloor+ \\
& c_{m} \cos \alpha \cdot \cos \beta\left[\dot{y}_{p}-\dot{y}_{g}+\dot{\theta}_{p z} R_{p}+\dot{\theta}_{g z} R_{g}-\dot{e}_{y}\right]
\end{aligned}
$$

The axial dynamic meshing force called $F_{z}$ is:

$$
\begin{aligned}
& F_{z}=k_{m z} f\left(z_{s}\right)+c_{m z} \dot{z}_{s}=k_{h} \cos \alpha \sin \beta \cdot f\left\lfloor z_{p}-z_{g}+\left(y_{p}+\theta_{p z} R_{p}-y_{g}+\theta_{p z} R_{g}\right) \tan \beta-e_{z}\right\rfloor+ \\
& c_{m} \cos \alpha \sin \beta\left[\dot{z}_{p}-\dot{z}_{g}+\left(\dot{y}_{p}+\dot{\theta}_{p z} R_{p}-\dot{y}_{g}+\dot{\theta}_{g z} R_{g}\right) \tan \beta-\dot{e}_{z}\right]
\end{aligned}
$$

The model equations set of helical gear driven system based on the newton second law is:

$$
\left\{\begin{array}{l}
m_{p} \ddot{x}_{p}+c_{p x} \dot{x}_{p}+k_{p x} f\left(x_{p}\right)=-F_{x} \\
m_{p} \ddot{y}_{p}+c_{p y} \dot{y}_{p}+k_{p y} f\left(y_{p}\right)=-F_{y} \\
m_{p} \ddot{z}_{p}+c_{p z} \dot{z}_{p}+k_{p z} f\left(z_{p}\right)=-F_{z} \\
J_{p y} \ddot{\theta}_{p y}+c_{p \theta y} \dot{\theta}_{p y} R_{p}+k_{p \theta y} \theta_{p y} R_{p}=-F_{z} R_{p} \\
J_{p z} \ddot{\theta}_{p z}=T_{p}-F_{y} R_{p} \\
m_{g} \ddot{x}_{g}+c_{g x} \dot{x}_{g}+k_{g x} f\left(x_{g}\right)=F_{x} \\
m_{g} \ddot{y}_{g}+c_{g y} \dot{y}_{g}+k_{g y} f\left(y_{g}\right)=F_{y} \\
m_{g} \ddot{z}_{g}+c_{g z} \dot{z}_{g}+k_{g z} f\left(z_{g}\right)=F_{z} \\
J_{g y} \ddot{\theta}_{g y}+c_{g \theta y} \dot{\theta}_{g y} R_{g}+k_{g \theta y} \theta_{g y} R_{g}=-F_{z} R_{g} \\
J_{g z} \ddot{\theta}_{g z}=-T_{g}+F_{y} R_{g}
\end{array}\right.
$$

Where, $R_{i}(i=p, g)$ are respectively the radius of base circle of driving gear and driven gear; $m_{i}(i=p, g), J_{i j}(i=p, g ; j=y, z)$ are respectively the mass and rotary inertia of driving gear and driven gear; $c_{i j}(i=p, g ; j=x, y, z), k_{i j}(i=p, g ; j=x, y, z)$ are respectively the supporting damping and supporting stiffness of driving gear and driven gear; $c_{i \theta y}(i=p, g), k_{i \theta y}(i=p, g)$ are respectively the damping and stiffness of torsion pendulum; $f\left(i_{j}\right)(i=x, y, z ; j=p, g, s)$ is the backlash nonlinearity function.

$b_{5}$ is the half backlash; $b_{1}$ and $b_{2}$ are respectively the half radial internal clearance; $2 b_{3}$ and $2 b_{4}$ are respectively the axial internal clearance; There are:

$$
\begin{aligned}
& f\left(x_{p}\right)=\left\{\begin{array}{ll}
x_{p}-b_{1} & x_{p}>b_{1} \\
0 & \left|x_{p}\right| \leq b_{1} \\
x_{p}+b_{1} & x_{p}<-b_{1}
\end{array}, f\left(y_{p}\right)=\left\{\begin{array}{ll}
y_{p}-b_{1} & y_{p}>b_{1} \\
0 & \left|y_{p}\right| \leq b_{1} \\
y_{p}+b_{1} & y_{p}<-b_{1}
\end{array}, f\left(z_{p}\right)= \begin{cases}z_{p}-b_{3} & z_{p}>b_{3} \\
0 & \left|z_{p}\right| \leq b_{3} \\
z_{p}+b_{3} & z_{p}<-b_{3}\end{cases} \right.\right. \\
& f\left(x_{g}\right)=\left\{\begin{array}{ll}
x_{g}-b_{2} & x_{g}>b_{2} \\
0 & \left|x_{g}\right| \leq b_{2} \\
x_{g}+b_{2} & x_{g}<-b_{2}
\end{array}, f\left(y_{g}\right)=\left\{\begin{array}{ll}
y_{g}-b_{2} & y_{g}>b_{2} \\
0 & \left|y_{g}\right| \leq b_{2} \\
y_{g}+b_{2} & y_{g}<-b_{2}
\end{array}, f\left(z_{g}\right)= \begin{cases}z_{g}-b_{4} & z_{g}>b_{4} \\
0 & \left|z_{g}\right| \leq b_{4} \\
z_{g}+b_{4} & z_{g}<-b_{4}\end{cases} \right.\right.
\end{aligned}
$$




$$
f\left(x_{s}\right)=\left\{\begin{array}{ll}
x_{s}-b_{5} & x_{s}>b_{5} \\
0 & \left|x_{s}\right| \leq b_{5} \\
x_{s}+b_{5} & x_{s}<-b_{5}
\end{array}, f\left(y_{s}\right)=\left\{\begin{array}{ll}
y_{s}-b_{5} & y_{s}>b_{5} \\
0 & \left|y_{s}\right| \leq b_{5} \\
y_{s}+b_{5} & y_{s}<-b_{5}
\end{array}, f\left(z_{s}\right)= \begin{cases}z_{s}-b_{5} & z_{s}>b_{5} \\
0 & \left|z_{s}\right| \leq b_{5} \\
z_{s}+b_{5} & z_{s}<-b_{5}\end{cases}\right.\right.
$$

The helical gear meshing has feature of "point - line - point". There is no phase step mutations in its time varying meshing stiffness of periodic changes. The time varying meshing stiffness of helical gear by Fourier transform method is:

$$
k_{h}(t)=k_{m}+\sum_{n=1}^{N}\left(a_{n} \cos n \omega_{0} t+b_{n} \sin n \omega_{0} t\right)
$$

Where, $\omega_{0}=2 \pi / T$ is the meshing fundamental frequency of gear pair, $T$ is the minimum positive period; $k_{m}$ is the average meshing stiffness of gear pair; $a_{n}$ and $b_{n}$ are the expansion coefficients of Fourier series, $n=1,2, \cdots, N$.

According to the 《Mechanical design manual》, $k_{m}$ is the meshing stiffness of ISO standards for helical gear, namely:

$$
k_{m}=\left(0.75 \varepsilon_{a}+0.25\right) C_{M} C_{R} C_{B} \cos \beta / q
$$

Where, $\varepsilon_{a}$ is the contact ratio.

$C_{B}$ is the coefficient of tooth profile, namely:

$$
C_{B}=\left[1+0.5\left(1.2-h_{f p} / m_{n}\right)\right] \times\left[1-0.02\left(20^{\circ}-\alpha_{n}\right)\right]
$$

$C_{R}$ is the structural coefficient of blank, solid gear: $C_{R}=1 ; C_{M}$ is the correction factor of theory, $C_{M}=0.8 ; q$ is the minimum value of tooth flexibility,namely:

$$
\begin{aligned}
& q=0.04723+\frac{0.15551}{z_{p}}+\frac{0.25791}{z_{g}}-0.00635 x_{p}-0.11654 \frac{x_{p}}{z_{p}}-0.00193 x_{g} \\
& -0.24188 \frac{x_{g}}{z_{g}}+0.00529 x_{p}^{2}+0.00182 x_{g}^{2}
\end{aligned}
$$

The meshing damping of tooth is[2]:

$$
c_{m}=2 \cdot \xi_{g} \sqrt{\frac{k_{m} m_{p} m_{g} R_{p}^{4} R_{g}^{4}}{2\left(m_{p} R_{p}^{4}+m_{g} R_{g}^{4}\right)}}
$$

Where, $\zeta_{g}$ is the meshing damping ratio, taking $[0.03,0.17]$.

Meshing error called $e(t)$, a displacement stimulus, is the elastic displacement in differential equation. $e(t)$ is:

$$
e(t)=e_{0}+e_{a} \sin (\omega t+\varphi)
$$

Where, $e_{0}$ is the constant, taking $0 ; e_{a}$ is the amplitude, taking $108 \mu \mathrm{m} ; \omega$ is the meshing frequency of gear pair; $\varphi$ is the phase angle.

\subsection{Model Dimensionless Processing.}

Order of magnitude difference between gear stiffness coefficient, damping ratio and vibratory response is bigger. Therefore, Eq. 5 needs dimensionless processing.

$\omega_{n}=\sqrt{k_{m} / m_{e}}$ is the natural frequency of helical gear pair, $m_{e}$ is the equivalent mass of gear, $b_{5}$ is the displacement scale, $\tau=t \cdot \omega_{n}$ is the dimensionless time. Therefore, the defined parameters are:

$p_{1}=x_{p} / b_{5}, p_{2}=y_{p} / b_{5}, p_{3}=z_{p} / b_{5}, p_{4}=R_{p} \theta_{p y} / b_{5}, p_{5}=R_{p} \theta_{p z} / b_{5}, p_{6}=x_{g} / b_{5}, p_{7}=y_{g} / b_{5}, p_{8}=z_{g} / b_{5}$, $p_{9}=R_{g} \theta_{g y} / b_{5}, p_{10}=R_{g} \theta_{g z} / b_{5}, p_{11}=x_{s} / b_{5}, p_{12}=y_{s} / b_{5}, p_{13}=z_{s} / b_{5}$

$\ddot{p}$ and $\dot{p}$ are respectively the first derivative and second derivative of $p$ to $\tau$, there are: 


$$
\begin{aligned}
& \dot{x}_{p}=\frac{d x_{p}}{d t}=\frac{d x_{p}}{d \tau} \cdot \frac{d \tau}{d t}=\frac{d\left(b_{5} \cdot p_{1}\right)}{d \tau} \cdot \frac{d\left(t \cdot \omega_{n}\right)}{d t}=b_{5} \cdot \dot{p}_{1} \cdot \omega_{n} \\
& \ddot{x}_{p}=\frac{d \dot{x}_{p}}{d t}=\frac{d\left(b_{5} \cdot \dot{p}_{1} \cdot \omega_{n}\right)}{d \tau} \cdot \frac{d \tau}{d t}=b_{5} \cdot \ddot{p}_{1} \cdot \omega_{n}^{2} \\
& \dot{\theta}_{p y}=\frac{d \theta_{p y}}{d t}=\frac{d \theta_{p y}}{d \tau} \cdot \frac{d \tau}{d t}=\frac{d\left(b_{5} \cdot p_{4} / R_{p}\right)}{d \tau} \cdot \frac{d\left(t \cdot \omega_{n}\right)}{d t}=\frac{b_{5} \cdot \dot{p}_{4} \cdot \omega_{n}}{R_{p}} \\
& \ddot{\theta}_{p y}=\frac{d \dot{\theta}_{p y}}{d t}=\frac{d\left(b_{5} \cdot \dot{p}_{4} \cdot \omega_{n} / R_{p}\right)}{d \tau} \cdot \frac{d \tau}{d t}=\frac{b_{5} \cdot \ddot{p}_{4} \cdot \omega_{n}^{2}}{R_{p}}
\end{aligned}
$$

The parameters of differential equation were transformed, getting the dimensionless equation, namely:

$$
\left\{\begin{array}{l}
\ddot{p}_{1}+\xi_{p x} \dot{p}_{1}+\eta_{p x} f\left(p_{1}\right)+\eta_{p m x} f\left(p_{11}\right)+\xi_{p m x} \dot{p}_{11}=0 \\
\ddot{p}_{2}+\xi_{p y} \dot{p}_{2}+\eta_{p y} f\left(p_{2}\right)+\eta_{p m y} f\left(p_{12}\right)+\xi_{p m p} \dot{p}_{12}=0 \\
\ddot{p}_{3}+\xi_{p z} \dot{p}_{3}+\eta_{p z} f\left(p_{3}\right)+\eta_{p m z} f\left(p_{13}\right)+\xi_{p m z} \dot{p}_{13}=0 \\
\ddot{p}_{4}+\xi_{p \theta y} \dot{p}_{4}+\eta_{p \theta_{y}} p_{4}+4 \eta_{p m z} f\left(p_{13}\right)+4 \xi_{p m m} \dot{p}_{13}=0 \\
\ddot{p}_{5}+2 \eta_{p m y} f\left(p_{12}\right)+2 \xi_{p m y} \dot{p}_{12}=g_{p} \\
\ddot{p}_{6}+\xi_{g x} \dot{p}_{6}+\eta_{g x} f\left(p_{6}\right)-\eta_{g m x} f\left(p_{11}\right)-\xi_{g m z} \dot{p}_{11}=0 \\
\ddot{p}_{7}+\xi_{g y} \dot{p}_{7}+\eta_{g y} f\left(p_{7}\right)-\eta_{g m y} f\left(p_{12}\right)-\xi_{g m z} \dot{p}_{12}=0 \\
\ddot{p}_{8}+\xi_{g z} \dot{p}_{8}+\eta_{g z} f\left(p_{8}\right)-\eta_{g m z} f\left(p_{13}\right)-\xi_{p m z} \dot{p}_{13}=0 \\
\ddot{p}_{9}+\xi_{g \theta y} \dot{p}_{9}+\eta_{g \theta_{y}} p_{9}+4 \eta_{g m z} f\left(p_{13}\right)+4 \xi_{g m z} \dot{p}_{13}=0 \\
\ddot{p}_{10}-2 \eta_{g m y} f\left(p_{12}\right)-2 \xi_{g m p} \dot{p}_{12}=-g_{g}
\end{array}\right.
$$

Where, the dimensionless damping coefficients are respectively $\xi_{p x}=\frac{c_{p x}}{m_{p} \omega_{n}}, \xi_{p y}=\frac{c_{p y}}{m_{p} \omega_{n}}$, $\xi_{p z}=\frac{c_{p z}}{m_{p} \omega_{n}}, \xi_{p \theta y}=\frac{2 c_{p x}\left(l_{1}+l_{2}\right)}{m_{p} R_{p} \omega_{n}}, \xi_{p m x}=\frac{c_{m} \sin \alpha}{m_{p} \omega_{n}}, \xi_{p m y}=\frac{c_{m} \cos \alpha \cos \beta}{m_{p} \omega_{n}}, \xi_{g x}=\frac{c_{g x}}{m_{g} \omega_{n}}, \xi_{p m z}=\frac{c_{m} \cos \alpha \sin \beta}{m_{p} \omega_{n}}, \xi_{g y}=\frac{c_{g y}}{m_{g} \omega_{n}}$, $\xi_{g z}=\frac{c_{g z}}{m_{g} \omega_{n}}, \xi_{g \theta_{y}}=\frac{2 c_{g x}\left(l_{3}+l_{4}\right)}{m_{g} R_{g} \omega_{n}}, \xi_{g m x}=\frac{c_{m} \sin \alpha}{m_{g} \omega_{n}}, \xi_{g m y}=\frac{c_{m} \cos \alpha \cos \beta}{m_{g} \omega_{n}}, \xi_{g m z}=\frac{c_{m} \cos \alpha \sin \beta}{m_{g} \omega_{n}}$. The dimensionless stiffness coefficients are respectively $\eta_{p x}=\frac{\omega_{p x}^{2}}{\omega_{n}^{2}}, \quad \eta_{p y}=\frac{\omega_{p y}^{2}}{\omega_{n}^{2}}, \quad \eta_{p z}=\frac{\omega_{p z}^{2}}{\omega_{n}^{2}}, \quad \eta_{p \theta y}=\frac{2 \omega_{p x}^{2}\left(l_{1}+l_{2}\right)}{R_{p} \omega_{n}^{2}}$, $\eta_{p m x}=[1+s \cdot \cos (\omega \tau)] \frac{m_{e} \sin \alpha}{m_{p}}, \eta_{g x}=\frac{\omega_{g x}^{2}}{\omega_{n}^{2}}, \eta_{g \theta y}=\frac{2 \omega_{g x}^{2}\left(l_{3}+l_{4}\right)}{R_{g} \omega_{n}^{2}}, \eta_{g y}=\frac{\omega_{g y}^{2}}{\omega_{n}^{2}}, \eta_{p m y}=[1+s \cdot \cos (\omega \tau)] \frac{m_{e} \cos \alpha \cos \beta}{m_{p}}, \eta_{g z}=\frac{\omega_{g z}^{2}}{\omega_{n}^{2}}$, $\eta_{p m z}=[1+s \cdot \cos (\omega \tau)] \frac{m_{e} \cos \alpha \sin \beta}{m_{p}}, \quad \eta_{g m x}=[1+s \cdot \cos (\omega \tau)] \frac{m_{e} \sin \alpha}{m_{g}}, \quad \eta_{g m y}=[1+s \cdot \cos (\omega \tau)] \frac{m_{e} \cos \alpha \cos \beta}{m_{g}}$, $\eta_{g m z}=[1+s \cdot \cos (\omega \tau)] \frac{m_{e} \cos \alpha \sin \beta}{m_{g}}, s$ is the coefficient, taking $0.1 . l_{1}, l_{2}, l_{3}$ and $l_{4}$ are respectively the distance from two supportings of driving gear ang driven gear to barycentric of gear. The rest of dimensionless coefficients are respectively $\omega_{p x}=\sqrt{k_{p x} / m_{p}}, \omega_{p y}=\sqrt{k_{p y} / m_{p}}, \omega_{p z}=\sqrt{k_{p z} / m_{p}}$, $\omega_{g x}=\sqrt{k_{g x} / m_{g}}, \omega_{g y}=\sqrt{k_{g y} / m_{g}}, \omega_{g z}=\sqrt{k_{g z} / m_{g}}, g_{p}=\frac{2 T_{p}}{m_{p} R_{p} b_{5} \omega_{n}^{2}}, g_{g}=\frac{2 T_{g}}{m_{g} R_{g} b_{5} \omega_{n}^{2}}, m_{e}=\frac{J_{p} J_{g}}{J_{p} R_{g}^{2}+J_{g} R_{p}^{2}}$.

\subsection{Solving Non-linear Dynamic Model of Gear Driven System.}

The specific parameters of helical gear are listed in Table 1. The displacement curve, speed curve, phase plane curve, poincare figure and FFT figrue are gained by fifth order variable step size and self adapted method (Runge-Kutta method). Due to the lack of space, when we took $n=3600 \mathrm{r} / \mathrm{min}$ (speed of rotation), $T=2600 \mathrm{~N} \cdot \mathrm{m}$ (twist moment), the dynamic responses of driving gear and driven gear along the $x$ direction are shown in Fig. 4 . 
Table 1. Parameters of helical gear driven system

\begin{tabular}{cccccc}
\hline $\begin{array}{c}\text { Number of } \\
\text { teeth } z_{1} / z_{2}\end{array}$ & $\begin{array}{c}\text { Modulus } \\
m_{n} / \mathrm{mm}\end{array}$ & $\begin{array}{c}\text { Helix angle } \\
\beta /{ }^{\circ}\end{array}$ & $\begin{array}{c}\text { Pressure } \\
\text { angle } \alpha /^{\circ}\end{array}$ & $\begin{array}{c}\text { Face } \\
\text { width } B / \mathrm{mm}\end{array}$ & Damping ratio coefficient \\
\hline $34 / 84$ & 6 & 17 & 25 & 70 & 0.08 \\
& \multicolumn{2}{c}{ bearing backlash of 1 gear $/ \mathrm{mm}$} & bearing backlash of 2 gear $/ \mathrm{mm}$ & $\begin{array}{c}\text { supporting stiffness } / \mathrm{N} / \mathrm{m} \\
\mathrm{m}\end{array}$ \\
backlash $/ \mathrm{mm}$ & axial & radial & axial & radial & $5.968 \times 10^{6}$ \\
& 0.148 & 0.107 & 0 & 0.075 & 5 \\
\hline
\end{tabular}

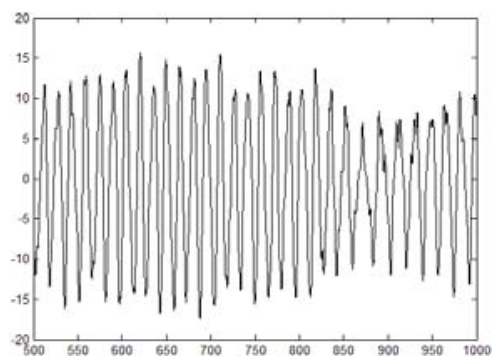

(a) Time - displacement response of driving gear

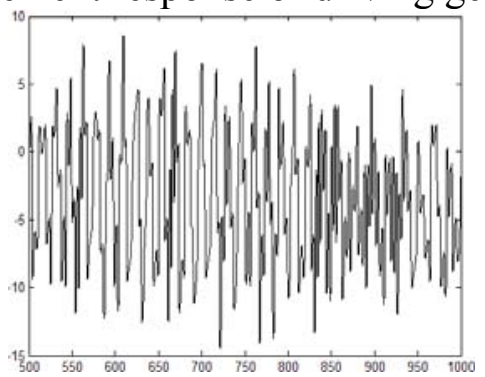

(c) Time - speed response of driving gear

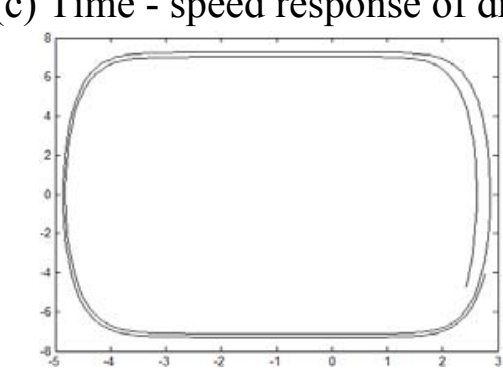

(e) Phase diagram of driving gear

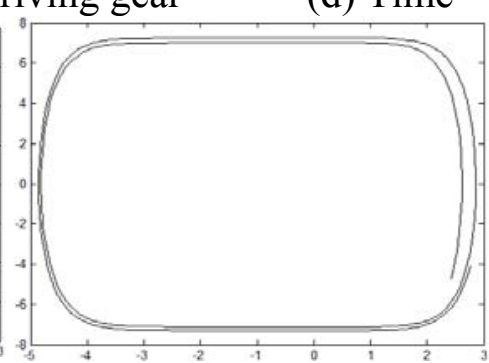

(f) Phase diagram of driven gear

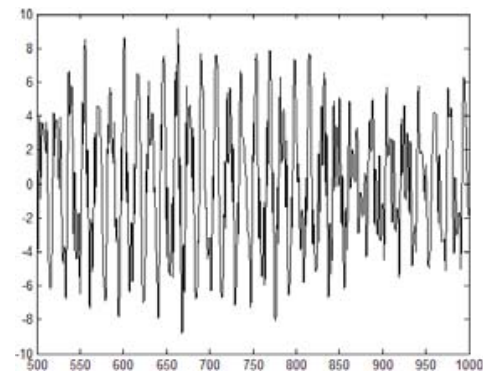

(b) Time - displacement response of driven gear

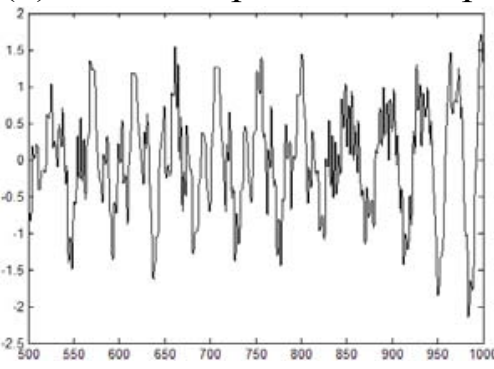

(d) Time - speed response of driven gear

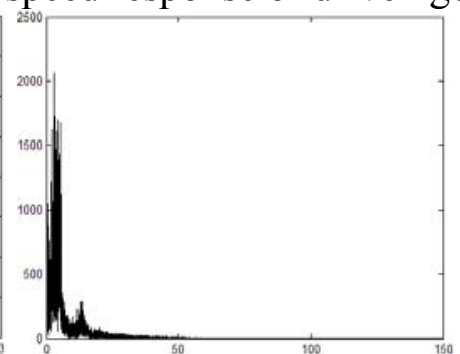

(g) FFT figure of driving gear

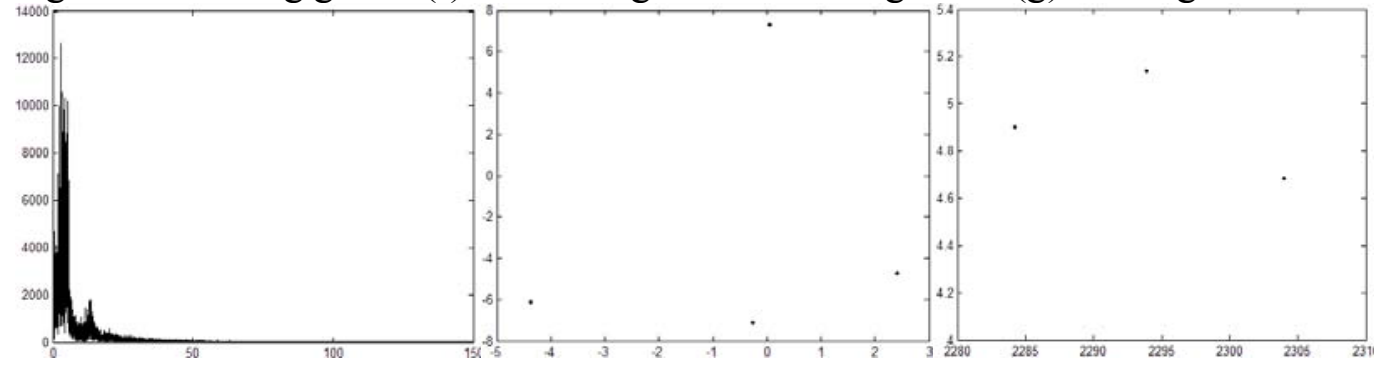

(h) FFT figure of driven gear (i) Poincare figure of driving gear (j) Poincare figure of driven gear

Fig. 4 Dynamic responses of the system along the $\mathrm{x}$ axis

The calculation shows that in the radial direction of $x$, the vibration amplitude of driving gear is larger than driven gear and the vibration of driving gear is more intense. The vibration speed of driven gear is more smooth than driving gear. The fluctuation amplitude and vibration frequency of driving gear is bigger than driven gear. The vibration rule of driving and driven gears is similar to monocyclic simple harmonic oscillation and the low-frequency signal of vibration occupies the main part. Phase diagrams show the unclosed curves with certain width that are aperiodic motion. There are four discrete points in the Poincare figure, therefore response is subharmonics trajectory of cycle 4. 


\section{A Non-linear Random Vibration Reliability Analysis for Multi Degree of Freedom Helical Gear Driven System}

We selected some parameters that have a larger impact on and are sensitive to the system. $B_{1}$ and $B_{2}$ are respectively the face width of driving and driven gears; $b_{5}$ is the backlash; row is the material density; $s$ is the coefficient; $n$ is the speed of rotation. The above parameters are random variables and set to meet normal distribution. The reliability distributed parameters are listed in Table 2.

Table 2 Random parameters' normal distributions of helical gear dynamic response

\begin{tabular}{ccccccc}
\hline Random parameters & $B_{1} / \mathrm{m}$ & $B_{2} / \mathrm{m}$ & $b_{5} / \mathrm{m}$ & $r o w / \mathrm{kg} / \mathrm{m}^{3}$ & $s$ & $\begin{array}{c}n / \mathrm{r} / \mathrm{mi} \\
\mathrm{n}\end{array}$ \\
\hline Equalizing value & $7 \times 10^{-2}$ & $7 \times 10^{-2}$ & $2 \times 10^{-5}$ & $7.85 \times 10^{3}$ & 0.1 & 4000 \\
Standard deviation & $(1 / 3) \times 10^{-3}$ & $(1 / 3) \times 10^{-3}$ & $(1 / 3) \times 10^{-8}$ & $1 / 3$ & $(1 / 3) \times 10^{-3}$ & $1 / 3$ \\
\hline
\end{tabular}

Considering the random error distribution of all variables, the oscillation equation of system is solved by fifth order variable step size and self adapted method (Runge-Kutta method) in each program loop and it is gained that the system response at any time in every degree of freedom. According to the flowchart of Fig. 1 , by $10^{4}$ random sampling, the instantaneous failure rate and instantaneous reliability of system are gained. At $t=200 \mathrm{~s}$, the probability distribution of vibratory response along $x$ axis is shown in Fig. 5 and the reliability results are listed in Table 3.
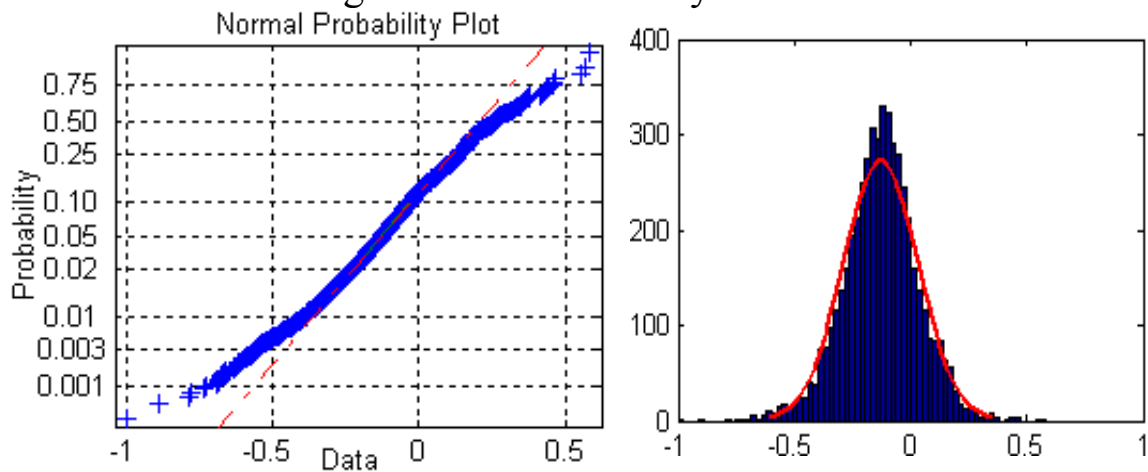

Fig. 5 Vibration reliability curves of the system along $\mathrm{x}$ axis at $\mathrm{t}=200 \mathrm{~s}$

Table 3 Random vibration reliabilities of the gear driven system at $\mathrm{t}=200 \mathrm{~s}$

\begin{tabular}{|c|c|c|c|c|c|}
\hline Variables & $x_{1}$ & $y_{1}$ & $z_{1}$ & $\theta_{1 y}$ & $\theta_{1 z}$ \\
\hline $\begin{array}{l}\text { Failure probability } \\
\qquad P_{f}\end{array}$ & 0.0148 & 0.0115 & 0.0197 & 0.0168 & 0.0128 \\
\hline $\begin{array}{l}\text { The instantaneous } \\
\text { reliability } P_{r}\end{array}$ & 0.09852 & 0.9885 & 0.9803 & 0.9832 & 0.9872 \\
\hline $\begin{array}{l}\text { Reliability indexs } \\
\beta\end{array}$ & 8.8057 & 9.7127 & 2.8025 & 2.8588 & 9.3206 \\
\hline $\begin{array}{l}\text { Regularities of } \\
\text { distribution }\end{array}$ & $\begin{array}{l}\text { Lognormal } \\
\text { distribution }\end{array}$ & $\begin{array}{l}\text { Lognormal } \\
\text { distribution }\end{array}$ & $\begin{array}{l}\text { Rayleigh } \\
\text { distribution }\end{array}$ & $\begin{array}{l}\text { Rayleigh } \\
\text { distribution }\end{array}$ & $\begin{array}{l}\text { Lognormal } \\
\text { distribution }\end{array}$ \\
\hline Variables & $x_{2}$ & $y_{2}$ & $z_{2}$ & $\theta_{2 y}$ & $\theta_{2 z}$ \\
\hline $\begin{array}{l}\text { Failure probability } \\
\qquad P_{f}\end{array}$ & 0.0146 & 0.0104 & 0.0184 & 0.0163 & 0.0138 \\
\hline $\begin{array}{l}\text { The instantaneous } \\
\text { reliability } P_{r}\end{array}$ & 0.9854 & 0.9896 & 0.9816 & 0.9837 & 0.9862 \\
\hline $\begin{array}{l}\text { Reliability indexs } \\
\beta\end{array}$ & 8.8532 & 10.0905 & 2.8268 & 8.4738 & 9.0515 \\
\hline $\begin{array}{l}\text { Regularities of } \\
\text { distribution }\end{array}$ & $\begin{array}{l}\text { Lognormal } \\
\text { distribution }\end{array}$ & $\begin{array}{l}\text { Lognormal } \\
\text { distribution }\end{array}$ & $\begin{array}{c}\text { Rayleigh } \\
\text { distribution }\end{array}$ & $\begin{array}{c}\text { Rayleigh } \\
\text { distribution }\end{array}$ & $\begin{array}{l}\text { Lognormal } \\
\text { distribution }\end{array}$ \\
\hline
\end{tabular}




\section{Summary}

The non-linear random vibration reliability analysis method for multi-degree of freedom helical gear driven system is established. This method can analyze effectively random vibration reliability of system at every time, which provides theoretical basis for system dynamic optimum design.

\section{Acknowledgments}

This work was supported by The National Natural Science Foundation of China (Grant No.E050402/51105187), General Project of Education Department in Liaoning Province (Grant No.L2014110), Innovation Team Project of Colleges and Universities in Liaoning Province (Grant No.LT2015014), Natural Science Foundation in Liaoning Province (Grant No.201602393), and Youth Science and Technology Talent Training Project in Anshan City (Grant No.0000077). The authors would like to thank the editors and anonymous reviewers for their useful comments.

\section{References}

[1] Dingguo Tang. Chen Guoming. Gear driven technology state and prospect. Journal of Mechanical Engineering. 1993; 29(5):35-42.

[2] Runfang Li. Wang Jianjun. Gear driven system dynamics-vibration, shock, and noise. Science press, 1997, p. 10-12.

[3] Neriya V, Bhat R B, Sanker T S. Coupled tensional flexural vibration of a geared shaft system using finite element analysis. The Shock and Vibration Bulletin. 1998;55:25-58.

[4] Runfang Li. Numerical simulation for inner dynamic excitation of gearing. Journal of Mechanical Transmission. 2001; 25(2): 1-3.

[5] Tengjiao Lin. Numerical simulation for inner dynamic excitation and system vibration response of speed increasing gearbox. Transactions of the Chinese Society of Agricultural Machinery. 2002;33(6): 20-22.

[6] Jing Wei. Dynamic analysis and effects of nonlinear factors of a helical gear pair based on improved coupling model of bending, torsion and swinging. Chinese Journal of Mechanical Engineering. 2011; 47(8):1-9. 